\title{
Effects of Intra-Aortic Balloon Pump Timing on Baroreflex Activities in a Closed-Loop Cardiovascular Hybrid Model
}

\author{
$* \ddagger$ Libera Fresiello, $\dagger$ Ashraf William Khir, *Arianna Di Molfetta, $\$$ Maciej Kozarski, \\ and *Gianfranco Ferrari \\ *Section of Rome, Institute of Clinical Physiology, National Council for Research, Rome, Italy; $†$ Institute of Bioengineering, \\ Brunel University, London, UK; and $\$$ Institute of Biocybernetics and Biomedical Engineering, Polish Academy of Science, \\ Warsaw, Poland
}

\begin{abstract}
Despite 50 years of research to assess the intraaortic balloon pump (IABP) effects on patients' hemodynamics, some issues related to the effects of this therapy are still not fully understood. One of these issues is the effect of IABP, its inflation timing and duration on peripheral circulation autonomic controls. This work provides a systematic analysis of IABP effects on baroreflex using a cardiovascular hybrid model, which consists of computational and hydraulic submodels. The work also included a baroreflex computational model that was connected to a hydraulic model with a $40-\mathrm{cm}^{3}$ balloon. The IABP was operated at different inflation trigger timings ( -0.14 to $0.31 \mathrm{~s})$ and inflation durations $(0.05-0.45 \mathrm{~s})$, with time of the dicrotic notch being taken as $t=0$. Baroreflex-dependent parametersafferent and efferent pathway activity, heart rate, periph-
\end{abstract}

eral resistance, and venous tone-were evaluated at each of the inflation trigger times and durations considered. Balloon early inflation ( $0.09 \mathrm{~s}$ before the dicrotic notch) with inflation duration of $0.25 \mathrm{~s}$ generated a maximum net increment of afferent pathway activity of $10 \%$, thus leading to a decrement of efferent sympathetic activity by $15.3 \%$ compared with baseline values. These times also resulted in a reduction in peripheral resistance and heart rate by 4 and $4.3 \%$ compared with baseline value. We conclude that optimum IABP triggering time results in positive effects on peripheral circulation autonomic controls. Conversely, if the balloon is not properly timed, peripheral resistance and heart rate may even increase, which could lead to detrimental outcomes. Key Words: Intra-aortic balloon pumpBaroreceptors-Balloon timing-Hybrid model.
The effects of the intra-aortic balloon pump (IABP) on the left ventricle (LV) and aortic hemodynamics have been the subject of investigation for 40 years $(1,2)$. Balloon inflation causes an increment of mean arterial blood pressure and of myocardial perfusion. Balloon deflation reduces afterload of the following beat, which causes a reduction of LV wall tension (1). The overall benefit is an increment of the ratio of myocardial oxygen supply to oxygen demand (1).

Few efforts have been spent so far to assess the effects of IABP on the autonomic control mechanisms that influence hemodynamics parameters such

doi:10.1111/j.1525-1594.2012.01540.x

Received May 2012; revised June 2012.

Address correspondence and reprint requests to Dr. Ashraf William Khir, Brunel Institute for Bioengineering, Brunel University, Uxbridge, Middlesex UB8 3PH, UK. E-mail: ashraf.khir@ brunel.ac.uk as peripheral resistance and heart rate (HR). The first of these mechanisms is to be activated in the presence of an external disturbance to the arterial baroreflex and acts as a negative feedback control loop of the systemic arterial pressure (3). This control is ruled by baroreceptors, a type of mechanoreceptor mainly located in the aortic arch and in the carotid sinuses of the left and right internal carotid arteries.

The premise of this work is that as baroreceptors are mainly located in an area very close to the descending aorta, the operation of an IABP can be expected to induce alterations in neurohumoral outflows and may thus affect the cardiovascular function. This premise was confirmed by a previous work (4) where it was demonstrated that the two peaks seen on the aortic waveform, which are caused by left ventricular ejection in systole and balloon inflation in diastole, are associated with two volleys of the afferent pathway, thus leading to a rise in baroreflex activity. Moreover, it has also been found that the 
increased stimulation during counterpulsation therapy produces reflex decreases in peripheral resistance and in HR through the efferent pathways (5).

Although IABP is mostly used in patients with severe heart failure, whose sympathetic nerve activity is increased (6), surprisingly only a few studies have examined the role of balloon inflation and deflation timing in relation to baroreflex activity. Barnea et al. (7) developed a numerical model of a cardiovascular system that included a local flow regulation and took into account the role of baroreflex during IABP assistance. Although that study provided fundamental understanding, the model did not include representation of afferent and efferent pathways and the IABP was represented through a computational model.

Therefore, the aim of this work is to study the baroreflex activity during IABP assistance in order to assess its potential beneficial effects on hemodynamic parameters such as peripheral resistance and HR, whose values are usually elevated in hypertensive and heart failure patients; reducing these parameters may contribute to reducing ventricular workload. To achieve our aim, we developed a hybrid model (computational and hydraulic) and we investigated the following: (i) the effects of IABP timing on baroreflex afferent and efferent pathways and (ii) how changes in baroreflex activity affect the hemodynamic parameters of a simulated assisted patient.

\section{MATERIALS AND METHODS}

The expression "hybrid model" indicates a special model whose structure is obtained by merging computational and physical submodels. The interaction between the computational and hydraulic submodels is assured through interfaces defined as "impedance transformers" (8-10). The model used in the present study is an extension of and is based largely on our previous hybrid model (9).

\section{The computational submodel}

The computational submodel was developed in LabVIEW 7.1 (National Instruments, Austin, TX, USA) environment and provides a lumped parameter representation of the pulmonary and systemic circulations as shown in Fig. 1. The pulmonary circulation is split into an arterial and venous part. Left and right ventricular contractions are described by a time varying elastance model (11), while the filling phase is represented by a sum of exponential technique (9). The systemic circulation is split into upper body, splanchnic, renal, and lower limb circulations, each of which includes both arterial and venous parts (12).
The aorta is split into two main sections, one is computational and the other is hydraulic (described below). The first part reproduces the behavior of the ascending aorta and of the aortic arch, providing the pressure signal driving the baroreflex computational submodel.

\section{The hydraulic submodel}

The second section of the aorta refers to the descending aorta and was implemented using a lumped compliance and a silicon rubber tube. The tube enables the insertion of a $40-\mathrm{cm}^{3}$ intra-aortic balloon driven by a Datascope System 96 (Datascope, Fairfield, NJ, USA), synchronized with the computational submodel by a transistor-transistor logic signal generated at the beginning of each ventricular contraction. The computational and hybrid submodels interact using two gear pumps and two pressure sensors. The pumps receive the signal from the computational submodel, and the pressure sensors provide information to the computational submodel. A more detailed description is provided in (9).

A Savitzky-Golay filter (13) is used for smoothing the pressure signals (Phyb1 and Phyb2) acquired from the hydraulic compliance and the impedance (Chyb and Zhyb) as shown in Fig. 1.

\section{The baroreflex computational representation}

The computational representation of the baroreflex used in this work is taken mainly after Ursino (14). The model provides a representation of the afferent pathway (Fas) and of both the sympathetic $(F e s)$ and vagal efferent pathways (Fev) in terms of spikes per second. The instantaneous value of pressure in the aortic arch block (Paa), as shown in Fig. 1, is used in a linear derivative first-order dynamic block as follows:

$$
\tau_{p} \cdot \frac{d P(t)}{d t}=P a a(t)+\tau_{z} \cdot \frac{d P a a(t)}{d t}-P(t)
$$

where $\tau_{p}$ and $\tau_{z}$ are the real pole and the real zero, respectively, of the first-order dynamics.

The output signal $P(t)$ is then used as an input to the calculation of Fas time course according to a sigmoidal characteristic as follows:

$$
\operatorname{Fas}(t)=\left[\frac{F a s_{\min }+F a s_{\max } \cdot e^{\left(\frac{P(t)-P a a_{S E T}}{k a}\right)}}{1+e^{\left(\frac{P(t)-P a a_{S E T}}{k a}\right)}}\right]
$$




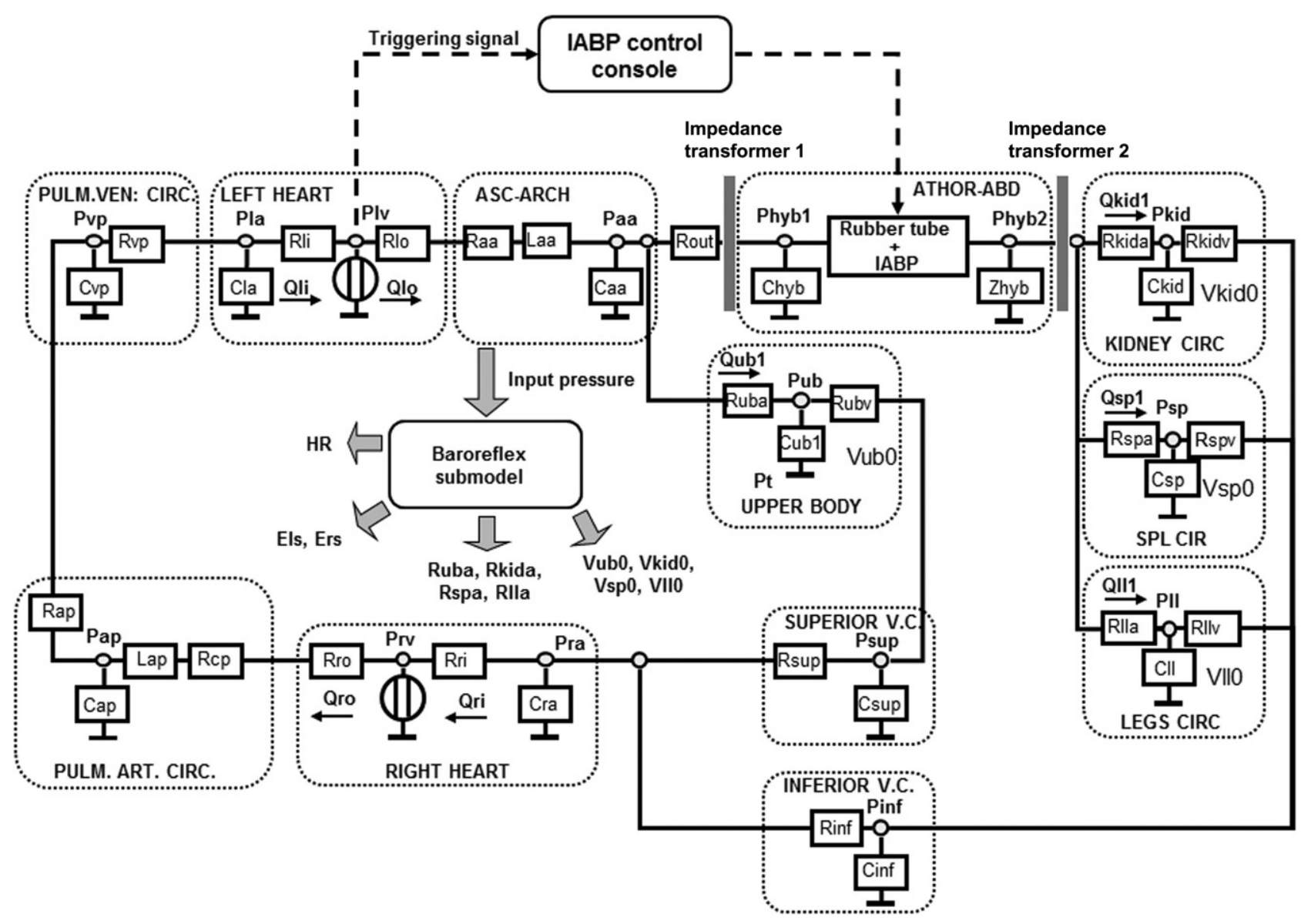

FIG. 1. Block diagram of the cardiovascular hybrid model. Impedance transformers 1 and 2 indicate the connections between the computational submodel and the hydraulic submodel. The first connection is realized between the ascending aorta (computational) and the descending aorta (hydraulic); the second interface is realized between the descending aorta and the lower body circulatory districts (computational). Zhyb and rubber tube blocks include resistive, capacitive, and inertial properties of connection tubing as well. From the left heart block, a transistor-transistor logic signal is generated and sent at the beginning of each ventricular contraction to the IABP control console. The baroreflex submodel is an additional computational module driven by the pressure Paa and controlling some hemodynamic parameters of the cardiovascular system.

where $\mathrm{Fas}_{\min }$ and $\mathrm{Fas}_{\max }$ are the lowest (2.52 spikes/s) and highest (47.78 spikes/s) values of the Fas signal, $P a a_{S E T}$ is the set-point aortic pressure, and $k a$ is a parameter regulating the slope of the sigmoidal characteristic close to its central point.

In a second block, Fes and Fev are obtained as a function of Fas as follows:

$$
F e s(t)=F e s_{\infty}+\left(F e s_{0}-F e s_{\infty}\right) \cdot e^{-k e s \cdot F a s(t)}
$$

$$
F e v(t)=\frac{F e v_{0}+F e v_{\infty} \cdot e^{\left(\frac{F a s(t)-F a s_{0}}{k e v}\right)}}{1+e^{\left(\frac{F a s(t)-F a s_{0}}{k e v}\right)}}
$$

where $\mathrm{Fes}_{\infty}, \mathrm{Fes}_{0}, \mathrm{kes}, \mathrm{Fev}_{0}, \mathrm{Fev}_{\infty}$, and kev are constant parameters. A list of all the parameters used in Eqs. $1-4$ is reported in Table 1. The final static function for

\begin{tabular}{|c|c|c|c|c|}
\hline Eq. & & & & \\
\hline (1) & $\tau_{p}=2.076 \mathrm{~s}$ & $\tau_{z}=6.37 \mathrm{~s}$ & & \\
\hline (2) & Fas $_{\min }=2.52 \mathrm{spikes} / \mathrm{s}$ & $F a s_{\max }=47.78$ spikes $/ \mathrm{s}$ & $k a=11.758 \mathrm{~mm} \mathrm{Hg}$ & \\
\hline (3) & $F e s_{\infty}=2.10$ spikes $/ \mathrm{s}$ & $F e s_{0}=16.11$ spikes $/ \mathrm{s}$ & $k e s=0.0675 \mathrm{~s}$ & \\
\hline (4) & $\mathrm{Fev}_{0}=3.2$ spikes $/ \mathrm{s}$ & $F e v_{\infty}=6.3$ spikes $/ \mathrm{s}$ & kev $=7.06$ spikes $/ \mathrm{s}$ & $F a s_{0}=25$ spikes $/ \mathrm{s}$ \\
\hline
\end{tabular}

TABLE 1. List of parameters used in Eqs. 1-4. Values are taken from (14) 
both sympathetic and vagal nerve $\left(s f_{s}, s f_{v}\right)$ is calculated in the third block.

The model is structured in such a way that it is possible to define a baseline condition in terms of a set-point aortic pressure $\left(P a a_{S E T}\right)$, systemic peripheral resistances, HR, left and right ventricular endsystolic elastance (Els and Ers, respectively), and venous tone (venous zero pressure filling volume). If the sensed $P a a$ is equal to the control pressure, the hemodynamic parameters assume their set-point values and no additional changes will occur. This was achieved by modifying the static function equations for both sympathetic $\left(s f_{H s}\right)$ and parasympathetic pathways $\left(s f_{H v}\right)$ taken from the baroreflex computational representation (14) as follows:

$$
\begin{gathered}
s f_{H s}(t)=G_{H s} \cdot\left(\left(\ln \left(F e s\left(t-D_{H s}\right)-2.65+1\right)\right)-1.1\right) \\
s f_{H v}(t)=G_{H v} \cdot\left(\operatorname{Fev}\left(t-D_{H v}\right)-4.66\right)
\end{gathered}
$$

where $G_{H s}\left(G_{H v}\right)$ is a gain factor and $D_{H s}\left(D_{H v}\right)$ is the time delay of the sympathetic (parasympathetic) activity.

According to Eqs. 5 and 6, both sympathetic and vagal static function values are zero for $P a a=P a a_{S E T}$. If $P a a$ is different from $P a a_{S E T}$, then the static functions will drive the first-order dynamics to change the parameters according to the following (14):

$$
\begin{gathered}
\frac{d \Delta H(t)}{d t}=\frac{\left(s f_{H s}(t)-\Delta H(t)\right)}{T_{H S}} \\
H(t)=\Delta H(t)+H_{S E T}
\end{gathered}
$$

where $\Delta H$ is the change of the generic hemodynamic variable under baroreflex control, $H_{S E T}$ is the setpoint value of the hemodynamic variable, and $T_{H s}$ is the time constant of the first-order dynamics. A similar equation is provided for the parasympathetic control of HR.

The sympathetic pathway influences HR, Els and Ers, arterial systemic resistances, and venous tones in the main circulatory districts (upper body, kidneys, splanchnic, and legs). Only HR is affected also by vagal efferent pathway activity.

\section{Hemodynamic parameter setting}

Experimental data from (15) were considered and used in order to characterize the cardiovascular model and to verify if the model was able to reproduce the hemodynamics before and after IABP onset. Experimental data refer to 12 heart failure Holstein calves (weight, $94 \pm 8 \mathrm{~kg}$ ) before and after IABP activation.
The hydraulic part of the model was characterized according to (9).

Values of all parameters, which are used to characterize the model, are provided in Table 2.

\section{Baroreflex parameter setting}

The baroreflex control is described by a firstorder dynamics and was defined according to literature data as follows: values of the first-order dynamics controlling HR for both sympathetic and parasympathetic pathways are taken from (14), values of the first-order dynamics controlling Els and Ers were defined considering a total increment over the whole baroreceptor sensitivity of $\sim 33 \%$ (14), and values of the first-order dynamics controlling peripheral resistance were defined considering a total increment of peripheral arterial resistances of $\sim 80 \%$ over the whole baroreceptor sensitivity range $(14,16-18)$. Finally, values of the first-order dynamics controlling venous tone were defined according to (19), so that the total splanchnic and extrasplanchnic venous tone increments over the whole baroreceptor sensitivity range were of 7.51 and $1.65 \mathrm{~mL} / \mathrm{kg}$, respectively.

A list of the parameters mentioned above, including time constants and time delays of the first-order dynamics, is reported in Table 3.

\section{Model verification procedure}

The first step of this work was to verify if the model was able to reproduce the hemodynamics of a heart failure condition (15). For this purpose, cardiovascular and baroreflex variables were manually set using the baseline condition of the cardiovascular and baroreflex models as shown in Tables 3 and 4, respectively. In this condition, $P a a=P a a_{S E T}$ so that the hemodynamic variables remain unchanged and the hemodynamic system is in an equilibrium condition.

The second step was to activate the IABP whose timing was set according to clinical guidelines. The model is left free to evolve toward a new equilibrium condition according to the effects of the balloon and the reaction of the baroreflex. In this phase of the validation, no manual changes of hemodynamic parameters were applied. The new hemodynamic condition was compared with the corresponding condition reported in (15).

\section{Varying the inflation trigger timing and the inflation duration}

The IABP performance was tested considering different inflation trigger timings and inflation durations. Starting from the baseline condition described so far, 
TABLE 2. List of cardiovascular parameters used in the simulations

\begin{tabular}{|c|c|c|c|c|}
\hline Cardiovascular parameters & Abbreviations & Units & Values & References \\
\hline Left/right ventricular rest volume & V01/V0r & $\mathrm{cm}^{3}$ & $70 / 5$ & $(15,9)$ \\
\hline Ventricular stiffness & $\mathrm{V}_{\text {stiffness }}$ & $\mathrm{mm} \mathrm{Hg} / \mathrm{cm}^{3}$ & 0.16 & (9) \\
\hline Left/right ventricular input resistance & Rli/Rri & $\mathrm{mm} \mathrm{Hg} \cdot \mathrm{s} / \mathrm{cm}^{3}$ & $0.02 / 0.02$ & (9) \\
\hline Left/right ventricular output resistance & Rlo/Rro & $\mathrm{mm} \mathrm{Hg} \cdot \mathrm{s} / \mathrm{cm}^{3}$ & $0.02 / 0.02$ & (9) \\
\hline Ascending aorta and aortic arch resistance & Raa & $\mathrm{mm} \mathrm{Hg} \cdot \mathrm{s} / \mathrm{cm}^{3}$ & 0.01 & (23) \\
\hline Ascending aorta and aortic arch inertance & Laa & $\mathrm{mm} \mathrm{Hg} \cdot \mathrm{s}^{2} / \mathrm{cm}^{3}$ & $5 \times 10^{-5}$ & (23) \\
\hline Ascending aorta and aortic arch compliance & Caa & $\mathrm{cm}^{3} / \mathrm{mm} \mathrm{Hg}$ & 0.5 & (9) \\
\hline Hybrid arterial compliance (mechanical) & Chyb & $\mathrm{cm}^{3} / \mathrm{mm} \mathrm{Hg}$ & 1.2 & (9) \\
\hline Silicon rubber tube compliance & Ctube & $\mathrm{cm}^{3} / \mathrm{mm} \mathrm{Hg}$ & 0.7 & (9) \\
\hline Upper body venous resistance & Rubv & $\mathrm{mm} \mathrm{Hg} \cdot \mathrm{s} / \mathrm{cm}^{3}$ & 0.23 & (12) \\
\hline Upper body compliance & $\mathrm{Cub}$ & $\mathrm{cm}^{3} / \mathrm{mm} \mathrm{Hg}$ & 8 & (12) \\
\hline Kidney venous resistance & Rkidv & $\mathrm{mm} \mathrm{Hg} \cdot \mathrm{s} / \mathrm{cm}^{3}$ & 0.3 & (12) \\
\hline Kidney compliance & Ckid & $\mathrm{cm}^{3} / \mathrm{mm} \mathrm{Hg}$ & 15 & (12) \\
\hline Splanchnic venous resistance & Rspv & $\mathrm{mm} \mathrm{Hg} \cdot \mathrm{s} / \mathrm{cm}^{3}$ & 0.18 & (12) \\
\hline Splanchnic compliance & Csp & $\mathrm{cm}^{3} / \mathrm{mm} \mathrm{Hg}$ & 55 & (12) \\
\hline Lower limbs venous resistance & Rllv & $\mathrm{mm} \mathrm{Hg} \cdot \mathrm{s} / \mathrm{cm}^{3}$ & 0.3 & (12) \\
\hline Lower limbs compliance & $\mathrm{Cll}$ & $\mathrm{cm}^{3} / \mathrm{mm} \mathrm{Hg}$ & 19 & (12) \\
\hline Superior vena cava resistance & Rsup & $\mathrm{mm} \mathrm{Hg} \cdot \mathrm{s} / \mathrm{cm}^{3}$ & 0.06 & (12) \\
\hline Superior vena cava compliance & Csup & $\mathrm{cm}^{3} / \mathrm{mm} \mathrm{Hg}$ & 15 & (12) \\
\hline Lower vena cava resistance & Rinf & $\mathrm{mm} \mathrm{Hg} \cdot \mathrm{s} / \mathrm{cm}^{3}$ & 0.015 & (12) \\
\hline Lower vena cava compliance & Cinf & $\mathrm{cm}^{3} / \mathrm{mm} \mathrm{Hg}$ & 27 & (12) \\
\hline Pulmonary characteristic resistance & Rcp & $\mathrm{mm} \mathrm{Hg} \cdot \mathrm{s} / \mathrm{cm}^{3}$ & 0.003 & (9) \\
\hline Pulmonary arterial compliance & Cap & $\mathrm{cm}^{3} / \mathrm{mm} \mathrm{Hg}$ & 1 & (9) \\
\hline Pulmonary arterial resistance & Rap & $\mathrm{mm} \mathrm{Hg} \cdot \mathrm{s} / \mathrm{cm}^{3}$ & 0.075 & (9) \\
\hline Pulmonary inertance & Lap & $\mathrm{mm} \mathrm{Hg} \cdot \mathrm{s}^{2} / \mathrm{cm}^{3}$ & $4 \times 10^{-5}$ & (9) \\
\hline Pulmonary venous compliance & Cvp & $\mathrm{cm}^{3} / \mathrm{mm} \mathrm{Hg}$ & 5 & (9) \\
\hline Pulmonary venous resistance & Rvp & $\mathrm{mm} \mathrm{Hg} \cdot \mathrm{s} / \mathrm{cm}^{3}$ & 0.003 & (9) \\
\hline Weight & W & $\mathrm{kg}$ & 94 & (15) \\
\hline
\end{tabular}

the IABP was driven with different timing settings to study the effects on baroreflex activity. Inflation trigger timings were progressively increased at each experiment starting from -0.14 to $0.31 \mathrm{~s}$ with a step of $0.05 \mathrm{~s}$ (the dicrotic notch was taken as $t=0 \mathrm{~s}$ ). For each value of inflation trigger timing, the deflation timing was progressively increased to obtain a balloon inflation duration ranging from 0.05 to $0.45 \mathrm{~s}$ with a step of $0.05 \mathrm{~s}$.

\section{RESULTS}

\section{Model verification}

The verification of the model was performed by comparing the literature and simulated data of the following:

Afferent pathway (Fas): Fig. 2 shows a comparison between the simulated Fas before and during

TABLE 3. List of baroreflex parameters used for simulations

\begin{tabular}{|c|c|c|c|c|}
\hline Variable & Static gain & Time constant & Time delay & Set-point value \\
\hline $\begin{array}{l}\text { Heart rate/heart cycle } \\
\text { duration }\end{array}$ & $\begin{array}{l}G_{\mathrm{TCs}}=-0.13 \mathrm{~s} / v(14) \\
G_{\mathrm{TCv}}=0.09 \mathrm{~s} / \nu(14)\end{array}$ & $\begin{array}{l}T_{\mathrm{TCs}}=2 \mathrm{~s}(14) \\
T_{\mathrm{TCv}}=1.5 \mathrm{~s}(14)\end{array}$ & $\begin{array}{l}D_{\mathrm{TCs}}=2 \mathrm{~s}(14) \\
D_{\mathrm{TCv}}=0.2 \mathrm{~s}(14)\end{array}$ & $\mathrm{HR}_{\mathrm{SET}}=94(15)$ \\
\hline $\begin{array}{l}\text { Left ventricular end-systolic } \\
\text { elastance }\end{array}$ & $G_{\mathrm{ELVs}}=0.17 \mathrm{~mm} \mathrm{Hg} / \mathrm{cm}^{3} / \mathrm{v}$ & $T_{\mathrm{ELVs}}=8 \mathrm{~s}(14)$ & $D_{\mathrm{ELVs}}=2 \mathrm{~s}(14)$ & $\operatorname{Els}_{\mathrm{SET}}=1.3(15)$ \\
\hline $\begin{array}{l}\text { Right ventricular end-systolic } \\
\text { elastance }\end{array}$ & $G_{\mathrm{ERVs}}=0.13 \mathrm{~mm} \mathrm{Hg} / \mathrm{cm}^{3} / \mathrm{v}$ & $T_{\mathrm{ERVs}}=8 \mathrm{~s}(14)$ & $D_{\mathrm{ERVs}}=2 \mathrm{~s}(14)$ & $\operatorname{ErS}_{\mathrm{SET}}=1.01(9)$ \\
\hline Upper body arterial resistance & $G_{\text {Rubas }}=1.97 \mathrm{~mm} \mathrm{Hg} \cdot \mathrm{s} / \mathrm{cm}^{3} / \mathrm{v}$ & $T_{\text {Rubas }}=6 \mathrm{~s}(14)$ & $D_{\text {Rubs }}=2 \mathrm{~s}(14)$ & $R_{\mathrm{ubaSET}}=5.50 \mathrm{~mm} \mathrm{Hg} \cdot \mathrm{s} / \mathrm{cm}^{3}(15)$ \\
\hline Kidney arterial resistance & $G_{\text {Rkidas }}=2.07 \mathrm{~mm} \mathrm{Hg} \cdot \mathrm{s} / \mathrm{cm}^{3} / \mathrm{v}$ & $T_{\text {Rkidas }}=6 \mathrm{~s}(14)$ & $D_{\text {Rkids }}=2 \mathrm{~s}(14)$ & $R_{\text {kidaSET }}=5.79 \mathrm{~mm} \mathrm{Hg} \cdot \mathrm{s} / \mathrm{cm}^{3}(15)$ \\
\hline Splanchnic arterial resistance & $G_{\mathrm{Rspas}}=1.51 \mathrm{~mm} \mathrm{Hg} \cdot \mathrm{s} / \mathrm{cm}^{3} / \mathrm{v}$ & $T_{\mathrm{Rspas}}=6 \mathrm{~s}(14)$ & $D_{\text {Rsps }}=2 \mathrm{~s}(14)$ & $R_{\mathrm{spaSET}}=4.23 \mathrm{~mm} \mathrm{Hg} \cdot \mathrm{s} / \mathrm{cm}^{3}(15)$ \\
\hline Lower limbs arterial resistance & $G_{\text {Rllas }}=1.81 \mathrm{~mm} \mathrm{Hg} \cdot \mathrm{s} / \mathrm{cm}^{3} / \mathrm{v}$ & $T_{\text {Rllas }}=6 \mathrm{~s}(14)$ & $D_{\text {Rlls }}=2 \mathrm{~s}(14)$ & $R_{\text {llaSET }}=5.10 \mathrm{~mm} \mathrm{Hg} \cdot \mathrm{s} / \mathrm{cm}^{3}(15)$ \\
\hline Upper body venous tone & $G_{\text {Voubs }}=-35 \mathrm{~cm}^{3} / \mathrm{v}$ & $T_{\text {Voubs }}=20 \mathrm{~s}(14)$ & $D_{\text {V0ubs }}=5 \mathrm{~s}(14)$ & $V_{\text {OubSET }}=650 \mathrm{~cm}^{3}(12)$ \\
\hline Kidney venous tone & $G_{\mathrm{V} 0 \mathrm{kids}}=-8 \mathrm{~cm}^{3} / \mathrm{v}$ & $T_{\mathrm{V} 0 \mathrm{kids}}=20 \mathrm{~s}(14)$ & $D_{\mathrm{V} 0 \mathrm{kids}}=5 \mathrm{~s}(14)$ & $V_{\text {0kidSET }}=150 \mathrm{~cm}^{3}(12)$ \\
\hline Splanchnic venous tone & $G_{\mathrm{V} 0 \text { sps }}=-282 \mathrm{~cm}^{3} / \mathrm{v}$ & $T_{\mathrm{V} 0 \mathrm{sps}}=20 \mathrm{~s}(14)$ & $D_{\mathrm{V} 0 \text { sps }}=5 \mathrm{~s}(14)$ & $V_{0 \text { spSET }}=1300 \mathrm{~cm}^{3}(12)$ \\
\hline \multirow[t]{2}{*}{ Lower venous tone } & $G_{\mathrm{V} 01 \mathrm{ls}}=-19 \mathrm{~cm}^{3} / \mathrm{v}$ & $T_{\mathrm{V} 011 \mathrm{~s}}=20 \mathrm{~s}(14)$ & $D_{\mathrm{V} 01 \mathrm{~s}}=5 \mathrm{~s}(14)$ & $V_{\text {OIISET }}=350 \mathrm{~cm}^{3}(12)$ \\
\hline & $P a a_{S E T}=$ & $\mathrm{n} \mathrm{Hg}(15)$ & & \\
\hline
\end{tabular}

$\mathrm{v}$ indicates the neural efferent rate (spikes/s). 
TABLE 4. Comparison between literature data (15) and simulation results before and after IABP onset

\begin{tabular}{|c|c|c|c|c|c|c|c|}
\hline Variable & Name & $\begin{array}{l}\text { Literature } \\
\text { IABP off }\end{array}$ & $\begin{array}{l}\text { Simulation } \\
\text { IABP off }\end{array}$ & $\%$ error & $\begin{array}{l}\text { Literature } \\
\text { IABP on }\end{array}$ & $\begin{array}{l}\text { Simulation } \\
\text { IABP on }\end{array}$ & $\%$ error \\
\hline Mean systemic arterial pressure & Paa mean $(\mathrm{mm} \mathrm{Hg})$ & 57 & 56 & $1.7 \%$ & 61 & 56 & $8.2 \%$ \\
\hline End diastolic volume & $\operatorname{EDV}\left(\mathrm{cm}^{3}\right)$ & 143 & 140 & $2.1 \%$ & 137 & 138 & $-0.7 \%$ \\
\hline End systolic volume & $\operatorname{ESV}\left(\mathrm{cm}^{3}\right)$ & 122 & 116 & $4.9 \%$ & 114 & 113 & $0.9 \%$ \\
\hline End diastolic pressure & $\mathrm{EDP}(\mathrm{mm} \mathrm{Hg})$ & 14.1 & 11 & $22 \%$ & 12.8 & 10.1 & $21 \%$ \\
\hline End systolic pressure & $\mathrm{ESP}(\mathrm{mm} \mathrm{Hg})$ & 68 & 67.6 & $0.6 \%$ & 62 & 63 & $-1.6 \%$ \\
\hline Heart rate & $\mathrm{HR}(\mathrm{bpm})$ & 94 & 92 & $2.1 \%$ & 91 & 89 & $2.2 \%$ \\
\hline Systemic peripheral resistance & $\operatorname{Ras}\left(\mathrm{mm} \mathrm{Hg} \cdot \mathrm{s} / \mathrm{cm}^{3}\right)$ & 1.48 & 1.48 & $0 \%$ & 1.46 & 1.44 & $1.37 \%$ \\
\hline
\end{tabular}

Literature data at IABP off were used in order to set the baseline condition of the hybrid model. At first, total systemic resistance was estimated as Ras $=($ mean systemic pressure - central venous pressure $) /$ cardiac output, according to (15). Then, resistance values of each circulatory district were estimated partitioning Ras in order to assure a distribution of cardiac output as follows: upper body, $23 \%$; kidney, $22 \%$; splanchnic, $30 \%$; and lower body, $25 \%$ (12).

IABP operation. During IABP assistance, two volleys for baroreceptor activity appear at each cardiac cycle: the first one due to left ventricular systole and the second one due to balloon inflation. The overall effect is a net increment of Fas of $8 \%$ (26.3 vs. 24.4 spikes/s).

Efferent pathway (Fes): in general, the increment of Fas induces a decrement of Fes, which is an inhibition of sympathetic activity. During IABP assistance, Fes decreased in comparison to that before IABP operation by $8 \%$ (5.56 vs. 6.06 spikes/s).

Hemodynamic parameters: a comparison between literature (15) and simulated data of systemic arterial pressure, HR, and LV volumes at both pump on and pump off is reported in Table 4. According to results, the parameters under baroreflex control show the following trend: HR decreases (91 vs. $94 \mathrm{bpm}$ according to [15] and 89 vs. $92 \mathrm{bpm}$ according to simulated data) and peripheral resistance decreases (1.46 vs. $1.48 \mathrm{~mm} \mathrm{Hg} \cdot \mathrm{s} / \mathrm{cm}^{3}$ according to [15] and 1.44 vs. $1.48 \mathrm{~mm} \mathrm{Hg} \cdot \mathrm{s} / \mathrm{cm}^{3}$ according to simulated data).

\section{The effects of varying balloon timing}

The effects of varying the trigger timing of IABP on baroreflex activity were compared with a common baseline condition at IABP off, used for the validation of the model. All results were summarized in Fig. 3.

- Hemodynamic variables: mean Paa in panel A.

- Baroreflex variables: Fas in panel B (maximum increment of $13.1 \%$ : 27.6 vs. 24.4 spikes/s), Fes in panel C (maximum decrement of $15.3 \%: 5.13$ vs. 6.06 spikes/s), and $\mathrm{Fev}$ in panel D (maximum increment of $6.5 \%: 5.05$ vs. 4.74 spikes/s).

- Hemodynamic parameters: HR in panel E (maximum decrement of 4.3\%: 88 vs. $92 \mathrm{bpm}$ ) and Rspa in panel F (maximum decrement of $4 \%: 4.1$ vs. $\left.4.3 \mathrm{~mm} \mathrm{Hg} \cdot \mathrm{s} / \mathrm{cm}^{3}\right)$. Only results for one of the four peripheral resistances were reported, as they all showed the same trend.

Figure $3 \mathrm{~A}$ shows an additional result of mean Paa depicted as a dashed line. These values were collected using the hybrid model, during IABP assistance for different timings without the baroreflex control.

\section{Early inflation}

In order to test the effects of early inflation, the balloon was inflated $0.14,0.09$, and $0.04 \mathrm{~s}$ before the dicrotic notch. If we analyze the effects of early inflation, we notice that the balloon positively stimulates the baroreflex, causing a reflex decrease in peripheral resistance and HR. The best timing was obtained for an early inflation of $-0.09 \mathrm{~s}$ with a Fas increment of $13.1 \%$ (27.6 vs. 24.4 spikes/s), Fes decrement of $11 \%$ (5.45 vs. $6.06 \mathrm{spikes} / \mathrm{s}), \mathrm{Fev}$ increment of $6.5 \%$ (5.05 vs. 4.74 spikes/s), Rspa decrement of $3.3 \%$ (4.15 vs. $4.3 \mathrm{~mm} \mathrm{Hg} \cdot \mathrm{s} / \mathrm{cm}^{3}$ ), HR decrement of $4.3 \%$ (88 vs. $92 \mathrm{bpm}$ ), and venous tone increment of $34 \mathrm{~cm}^{3}$.

\section{Late inflation}

In order to test the effects of late inflation, the balloon was inflated $0.01,0.06,0.11,0.16$, and $0.21 \mathrm{~s}$ after the dicrotic notch. If we analyze the effects of an inflation delay ranging from 0.06 to $0.21 \mathrm{~s}$, we notice that the balloon negatively stimulates the baroreflex system (for any value of inflation duration). Results show that Fas decreases (maximum decrement $11.4 \%, 22.23$ vs. 25.1 spikes/s), Fes increases (maximum increment of $11.7 \%, 6.68$ vs. 5.98 spikes/s), Fev decreases (maximum decrement of $7.1 \%, 4.44$ vs. 4.78 spikes/s), HR increases (7.6\%, 99 vs. 92 bpm), Rspa increases $\left(11 \%, 4.55\right.$ vs. $\left.4.1 \mathrm{~mm} \mathrm{Hg} \cdot \mathrm{s} / \mathrm{cm}^{3}\right)$, and venous tone decreases $\left(-50 \mathrm{~cm}^{3}\right)$.

\section{Inflation duration}

For each value of inflation timing set during the experiments, the deflation trigger timing was 

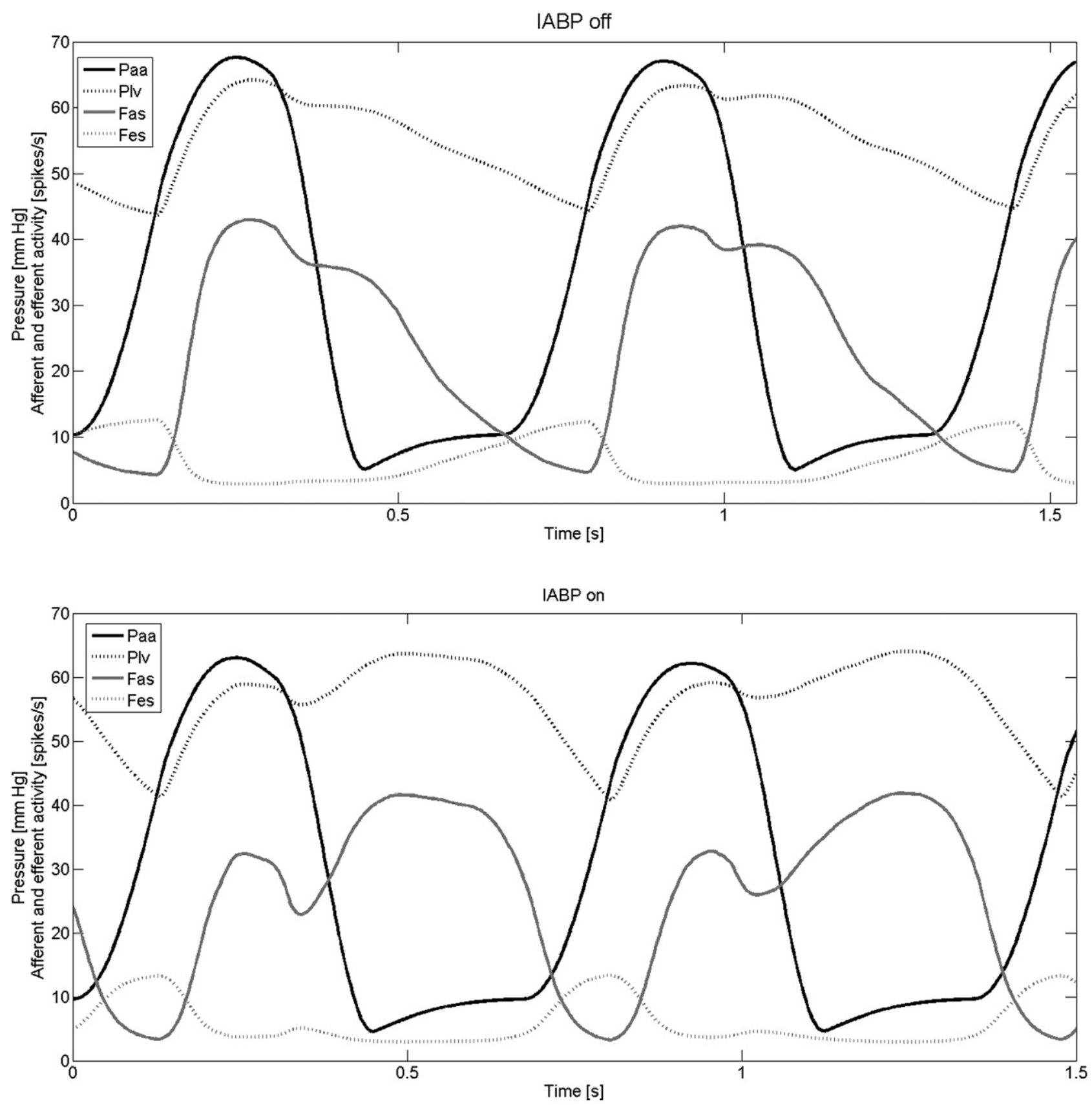

FIG. 2. Systemic arterial pressure (Paa), left ventricular pressure (PlV), and baroreflex activity waveforms. Upper (lower) panel refers to hemodynamic condition at IABP off (on).

changed progressively in order to test several IABP filling durations. Results show that the best inflation duration depends on the inflation trigger timing: that is, for an early inflation of $-0.09 \mathrm{~s}$ the best baroreflex stimulation was obtained for an inflation duration of $0.25 \mathrm{~s}$, for an early inflation of $-0.04 \mathrm{~s}$ the best results were observed for an inflation duration of $0.2 \mathrm{~s}$, and for an inflation timing at the dicrotic notch (inflation delay of $0.01 \mathrm{~s}$ ) the best inflation duration is $0.15 \mathrm{~s}$. However, for a late inflation, the baroreflex is negatively stimulated, no matter which value of inflation duration is set.

\section{DISCUSSION}

IABP modeling can be performed by connecting the IABP to a circulatory hydraulic model (20-22) or connecting a computational circulatory model to a 

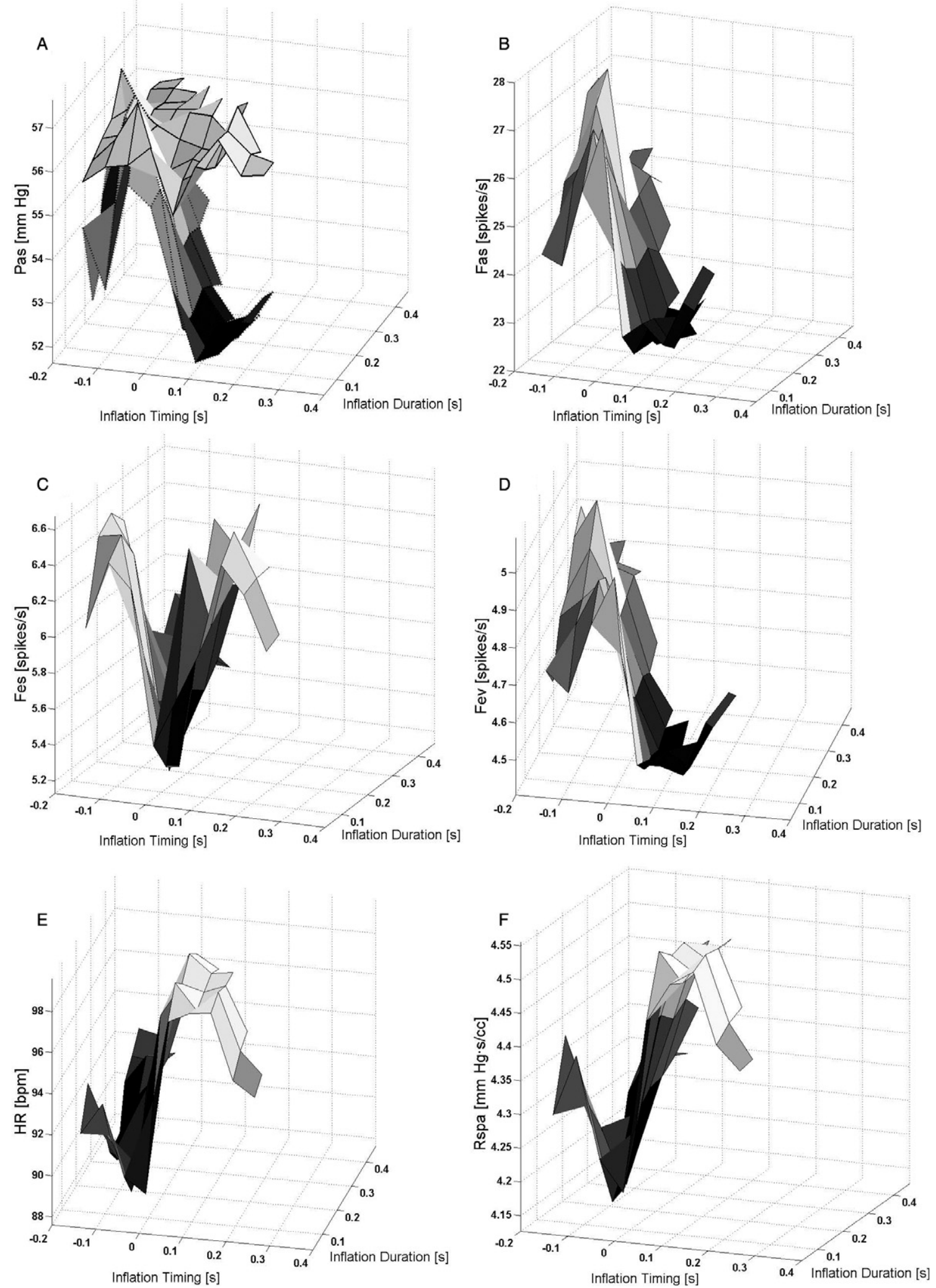

FIG. 3. Experimental results obtained with the hybrid model running the balloon with different inflation timings and inflation durations: (A) values of mean $\mathrm{Paa}$ (continuous [dashed] line refers to results with [without] baroreflex model), (B) baroreflex afferent activity, (C) efferent sympathetic activity, (D) efferent vagal activity, (E) heart rate, and (F) splanchnic arterial resistance. 
computational model of the balloon itself (23). In the present work, we advance the hybrid model of the cardiovascular system (9), by including a specific computational representation for baroreflex, and examine its activity in connection to the IABP.

The choice of a hybrid model is an attempt to overcome the difficulty of simulating the operation of a real cardiac assist device in a computational circulatory model. The computational submodel assures a good flexibility and a low cost of the hybrid system, while the physical submodel provides an interface for the specific device to be tested (8). Hybrid models behave very similar to the corresponding computational model and exhibit all the advantages of computational models in terms of reproducibility and repeatability of the experiments. This is a fundamental feature when reproducing the behavior of complex biological system depending on several variables.

\section{Model verification}

Normann and Kennedy found that the baroreflex activities are influenced by the operation of IABP (4). In particular, the counterpulsation effect provides a stimulation for the baroreceptors within the diastolic phase of the cardiac cycle. The overall effect is a net increment of the afferent activity. These observations are in good agreement with the results presented in the present work where the hybrid model reproduced qualitatively the trend previously observed in vivo (4). Further, Tokunaga et al. (6) investigated the effect of IABP on renal sympathetic nerve stimulation and reported a reduction of $2 \%$ for Fes in healthy animals and $9 \%$ in a heart failure animal model, which are in good agreement with our results of the present work where we report a $8 \%$ reduction of Fes during IABP assistance. Furthermore, the hemodynamic results of the hybrid model are generally in accordance with literature results as seen in Table 4. Although there are some differences in LV filling pressure and volume, probably due to the need of a better characterization of the ventricular diastolic function $\left(\mathrm{V}_{\text {stiffness }}\right)$, our main goal to ascertain that this hybrid model was able to reproduce the trend of these variables switching between IABP off and on modalities was achieved.

\section{Baroreflex variables}

Figure 3A shows that mean Paa in the presence of baroreflex varies less than mean $P a a$ data acquired using the same hydraulic submodel without a baroreflex control. This is a confirmation that the baroreflex computational representation is able to accomplish its main task: to keep blood pressure as close as possible to a set-point value $\left(\mathrm{Paa}_{S E T}\right)$ despite an external stimulus (in this case IABP onset).

The influence of IABP inflation trigger timings and of balloon inflation duration on baroreflex activity is usefully presented in Fig. 3B. Bearing in mind the time of the dicrotic notch corresponds to $t=0 \mathrm{~s}$, for inflation timing, an early inflation of $-0.09 \mathrm{~s}$ and an inflation duration of $0.25 \mathrm{~s}$ resulted in a net increment of Fas.

Fes and Fev trends, both ruled by Fas signal, are exactly the opposite as shown in Fig. 3C,D: when Fas increases, $\mathrm{Fes}$ decreases ( $\mathrm{Fev}$ increases) and vice versa. For a late inflation, the positive effects of baroreflex stimulation are reduced: in an inflation delay $>0.01 \mathrm{~s}$, the effects of IABP on baroreflex are even negative. This is probably due to an early inflation of the balloon prolonging the duration of baroreceptor stimulation started during the ventricular systole. A late inflation results in the loss of the second increase in diastolic aortic pressure and baroreceptors do not benefit from the counterpulsation effect.

Figure 3E shows that HR is influenced by both efferent pathways: Fes provokes an increment while $\mathrm{Fev}$ provokes a decrement of HR. If the balloon is properly timed, Fas increases, HR decreases, while late inflation results in an increase in HR.

Furthermore, splanchnic arterial resistances are reduced if the balloon is properly timed (Fas increases), as shown in Fig. 3F (other arterial resistances show a similar trend). Inflation delays $>0.01 \mathrm{~s}$ cause even an increase in peripheral arterial resistances.

Venous tone was also affected by baroreflex for the same timing mentioned above. Proper IABP timing causes an increment of $\sim 34 \mathrm{~cm}^{3}$ of the total venous tone observed, while for higher inflation delays venous tone decreased to $\sim 50 \mathrm{~cm}^{3}$ (a sympathetic inhibition corresponds to a decrement of zero pressure filling volume and vice versa).

Both left and right ventricular contractility were slightly affected (Els $=1.29-1.34$ and Ers $=1.00$ $1.04 \mathrm{~mm} \mathrm{Hg} / \mathrm{cm}^{3}$ ) and this is in accordance with previously published observations by Kawaguchi et al. (15).

According to the results mentioned so far, some additional considerations should be done:

- Baroreflex activity can strongly vary among different patients. As a consequence, nervous and hemodynamic parameter variations due to IABP can be more or less important. Previous work (6) has shown that in heart failure animals, because of an increased sympathetic activity, the positive effects 
of IABP on baroreflex were even higher in comparison to healthy animals.

- The aim of this work is not to provide a definitive indication about the best IABP timings, as this is still an open question (24-26) involving several variables (hemodynamic, energetic, etc.). The present work highlights which are the effects of IABP counterpulsation on baroreflex and which are the possible negative effects if balloon inflation trigger is not properly timed. Results show that small changes or alterations of the IABP timing can lead to a different stimulation of the baroreflex activity, thus leading the hemodynamic parameters to change differently.

It is important to note that the IABP operation does not actively empty the LV in comparison with other modalities of temporary cardiac assist devices such as rotary blood pumps. However, the IABP overall hemodynamic effects/benefits appear to be at least equal to that of intra-aortic rotary blood pumps. For example, a clinical trial aimed at comparing the IABP and Impella was recently halted due to futility (27). This contrast between these two modalities of temporary LV mechanical assistance has been recently discussed (28), and it was suggested that the IABP operation may induce a secondary effect that is not yet adequately appreciated or studied, involving the role of baroreceptors. Hence, we believe that the current work may shed more light on the mechanism underlying the positive effects of balloon counterpulsation.

Clinical IABP timing is set in order to inflate the balloon just before the dicrotic notch (before the closure of the aortic valve). Balloon deflation is usually set to take place toward the end of diastolic phase (conventional timing) or during the preejection phase or even early systolic ejection (according to real-time timing [29]).

According to our results, an early inflation (before the dicrotic notch) could assure a best baroreflex stimulation due to the balloon activity. Balloon inflation duration seems to be strictly dependent on early inflation timing with the earliest inflation triggering time resulting in the longest balloon inflation duration. Conversely, late inflation results in the balloon negatively stimulating the baroreflex regardless of the length of the inflation duration.

\section{CONCLUSIONS}

The hybrid model presented in this work adequately simulates the baroreflex activities and demonstrates its dependence on intra-aortic balloon pump operation. IAB inflation timings used in current clinical practice stimulate baroreflex as it generates a second pressure peak during diastole, thus increasing baroreceptor activity and causing a sympathetic inhibition. The increase in baroreceptor activity and the sympathetic inhibition result in a decrement of peripheral resistance and HR and an increment of venous tone. In our experiments, these effects can be maximized by triggering the IABP inflation before the dicrotic notch (inflation $-0.09 \mathrm{~s}$ ) with an inflation duration of $0.25 \mathrm{~s}$. Balloon triggering time of inflation after the dicrotic notch inhibits the positive stimulation of the baroreflex, which leads to a lack of increment or even a decrement in Fas, resulting in an increase of HR and peripheral resistance and a decrease in venous tone. Correct balloon timing of inflation and duration is critical to obtain the full benefits of balloon counterpulsation.

Acknowledgment: This work was partly supported by EU project SensorART (Grant number: 248763).

\section{Conflict of Interest: None.}

Author contributions: Libera Fresiello: Concept/ design, data analysis/interpretation, drafting article, and hybrid model development.

Ashraf W. Khir: Data analysis/interpretation and critical revision of article.

Arianna Di Molfetta: Critical revision of article and ventricular model development.

Maciej Kozarski: Critical revision of article and hybrid model development.

Gianfranco Ferrari: Critical revision of article, data analysis/interpretation, and hybrid model development.

\section{REFERENCES}

1. Santa-Cruz RA, Cohen MG, Ohman EM. Aortic counterpulsation: a review of the hemodynamic effects and indications for use. Catheter Cardiovasc Interv 2006;67:68-77.

2. Bolooki H. Pulsatile assist device and counterpulsation. In: Bolooki $\mathrm{H}$, ed. Clinical Application of Intra-Aortic Balloon Pump. Mount Kisco, NY: Futura Publishing Co., 1984;13-8.

3. Guyton AC, Jones CE, Coleman T. Autonomic regulation of cardiac output. In: Guyton SC, Jones CE, Coleman T, eds. Circulatory Physiology: Cardiac Output and Its Regulation. Philadelphia, London, Toronto: W.B. Saunders Company, 1973;317-9.

4. Normann NA, Kennedy JH. Arterial baroreceptor responses to intraortic balloon assistance. J Surg Res 1971;11:396-400.

5. Feola M, Limet RR, Glick G. Direct and reflex vascular effects of intra-aortic balloon counterpulsation in dogs. Am J Physiol 1971;221:748-53.

6. Tokunaga S, Tominaga R, Nakano T, Fukae K, Takeshita A, Yasui $\mathrm{H}$. Effects of intra-aortic balloon pumping on renal 
sympathetic nerve activity and renal circulation in dogs. J Cardiovasc Surg (Torino) 2000;41:669-74.

7. Barnea O, Moore TW, Dubin SE, Jaron D. Cardiac energy considerations during intraaortic balloon pumping. IEEE Trans Biomed Eng 1990;37:170-81.

8. Kozarski M, Ferrari G, Zielinski K, et al. A new hybrid electronumerical model of the left ventricle. Comput Biol Med 2008;38:979-89.

9. Ferrari G, Khir AW, Fresiello L, Di Molfetta A, Kozarski M. Hybrid model analysis of intra-aortic balloon pump performance as a function of ventricular and circulatory parameters. Artif Organs 2011;35:902-11.

10. Ferrari G, Kozarski M, Gu YJ, et al. Application of a user friendly comprehensive circulatory model for hemodynamic and ventricular variables estimate. Int J Artif Organs 2008;31: 1043-54.

11. Sagawa K, Maughan L, Suga H, Sunagawa K. Chamber pressure-volume relation versus muscle tension-length relaxation. In: Sagawa K, Maughan L, Suga H, Sunagawa K, eds. Cardiac Contraction and the Pressure-Volume Relationship. New York: Oxford University Press, 1988;61-94.

12. Heldt T, Shim EB, Kamm RD, Mark RG. Computational modeling of cardiovascular response to orthostatic stress. $J$ Appl Physiol 2002;92:1239-54.

13. Savitzky A, Golay MJE. Smoothing and differentiation of data by simplified least squares procedures. Anal Chem 1964;36: 1627-39.

14. Ursino M. Interaction between carotid baroregulation and the pulsating heart: a mathematical model. Am J Physiol 1998;275: 1733-47.

15. Kawaguchi O, Pae WE, Daily BB, Pierce WS. Ventriculoarterial coupling with intra-aortic balloon pump in acute ischemic heart failure. J Thorac Cardiovasc Surg 1999;117:164-71.

16. Cox RH, Bagshaw RJ. Baroreceptor reflex control of arterial hemodynamics in the dog. Circ Res 1975;37:772-86.

17. Angell James JE, Daly Mde B. Effects of graded pulsatile pressure on the reflex vasomotor responses elicited by changes of mean pressure in the perfused carotid sinus-aortic arch regions of the dog. J Physiol 1971;214:51-64.

18. Shoukas AA, Brunner MC. Epinephrine and the carotid sinus baroreceptor reflex. Influence on capacitive and resistive properties of the total systemic vascular bed of the dog. Circ Res 1980;47:249-57.

19. Brunner MJ, Shoukas AA, Macanespie CL. The effect of the carotid sinus baroreceptor reflex on blood flow and volume redistribution in the total systemic vascular bed of the dog. Circ Res 1981;48:274-85.

20. Vandenberghe S, Segers P, Josemans H, Van Loon J-P, Rakhorst G, Verdonck P. In vitro assessment of the unloading and perfusion capacities of the PUCA II and the IABP. Perfusion 2004;19:25-32.

21. Kolyva C, Biglino G, Pepper JR, Khir AW. A mock circulatory system with physiological distribution of terminal resistance and compliance: application for testing the intra-aortic balloon pump. Artif Organs 2012;36:E62-70.

22. Schampaert S, Van't Veer M, Van de Vosse FN, Pijls NHJ, De Mol BA, Rutten MCM. In vitro comparison of support capabilities of intra-aortic balloon pump and impella 2.5 left percutaneous. Artif Organs 2011;35:893-901.

23. Ferrari G, Kozarski M, Zieliński K, et al. A modular computational circulatory model applicable to VAD testing and training. J Artif Organs 2011;15:32-43.

24. Kern M, Aquirre F, Caracciolo E, et al. Hemodynamic effects of new intra-aortic balloon counterpulsation timing methods in patients: a multicenter evaluation. Am Heart J 1999;137: 1129-36.

25. Schreuder J, Maisano F, Donelli A, et al. Beat-to-beat effects of intra-aortic balloon pump timing on left ventricular performance in patients with low ejection fraction. Ann Thorac Surg 2005;79:872-80.

26. Schreuder J, Castiglioni A, Donelli A, et al. Automatic intraaortic balloon pump timing using an intrabeat dicrotic notch prediction algorithm. Ann Thorac Surg 2005;79:1017-22.

27. O'Neill W, et al. PROTECT II: a prospective, multicenter, randomized clinical trial of Intra-Aortic Balloon Pump versus Impella for hemodynamic support during high-risk PCI. ACC 2011:2131-8. Abstract.

28. Khir AW. Left ventricular temporary assistance: by blood propelling or counterpulsating? Artif Organs 2011;35:840-2.

29. Hanlon-Pena PM, Quaal SJ. Intra-aortic balloon pump timing. review of evidence supporting current practice. J Crit Care 2011;20:323-34. 